\title{
Oncogenic secretory clusterin in hepatocellular carcinoma: Expression at early staging and emerging molecular target
}

\author{
Wenjie Zheng ${ }^{1, *}$, Min Yao ${ }^{2, *}$, Qi Qian ${ }^{3, *}$, Wenli Sai ${ }^{1}$, Liwei Qiu ${ }^{1}$, Junling Yang ${ }^{1}$, Wei \\ $\mathbf{W u}^{1}$, Zhizhen Dong ${ }^{1,4}$ and Dengfu Yao ${ }^{1}$ \\ ${ }^{1}$ Research Center of Clinical Medicine, Affiliated Hospital of Nantong University, Nantong 226001, Jiangsu Province, China \\ ${ }^{2}$ Department of Immunology, Medical School of Nantong University, Nantong 226001, Jiangsu Province, China \\ ${ }^{3}$ Department of Oncology, Yancheng $1^{\text {st }}$ People's Hospital, Yancheng 224005, Jiangsu Province, China \\ ${ }^{4}$ Department of Diagnostics, Affiliated Hospital of Nantong University, Nantong 226001, Jiangsu Province, China \\ *These authors have contributed equally to this work \\ Correspondence to: Dengfu Yao, email: yaodf@ahnmc.com \\ Zhizhen Dong, email: dongzz@ahnmc.com \\ Keywords: hepatocellular carcinoma, secretory clusterin, prognosis, tumor stage, tumor growth \\ Received: September 20,2016 Accepted: November 18, $2016 \quad$ Published: November 29, 2016 \\ Copyright: Zheng et al. This is an open-access article distributed under the terms of the Creative Commons Attribution License 3.0 \\ (CC BY 3.0), which permits unrestricted use, distribution, and reproduction in any medium, provided the original author and source \\ are credited.
}

\section{ABSTRACT}

\begin{abstract}
Secretory clusterin (SCLU) is associated with hepatocellular carcinoma (HCC) progression by contributing to angiogenesis, chemoresistance, cell survival, and metastasis. However, the SCLU expression at early stage of HCC progression remains to be clarified. In this study, the alteration of SCLU oncogenicity was firstly evaluated in HCC- and their para-cancerous- tissues. The incidence of SCLU expression in HCC was significantly higher than that in their non-tumorous tissues at message RNA (mRNA) or protein level, gradually increasing with tumor-node-metastasis (TNM) staging. Abnormal sCLU expression was associated with the poor differentiation, TNM stage, and considered as an independent prognostic factor for HCC patients. Furthermore, silencing SCLU gene transcription inhibited the colony formation and proliferation of HCC cells, with decreasing phosphorylation level of AKT and GSK-3 $\beta$ in HCCLM3 cells in vitro and significantly suppressed the HCC xenograft growth in vivo, suggesting that SCLU with oncogenicity should be not only an early indicator but also novel potential molecular-targeted therapy for HCC.
\end{abstract}

\section{INTRODUCTION}

Hepatocellular carcinoma (HCC) is one of the most common malignant cancers and the $3^{\text {rd }}$ most frequent cause of cancer death worldwide [1-3]. Hepatitis B virus (HBV) or hepatitis $\mathrm{C}$ virus (HCV) infection along with alcohol and aflatoxin B1 intake are widely recognized as etiological agents in HCC [4-6]. Rapid progression, insensitivity to radiotherapy or chemotherapy, extensive metastasis and recurrence after surgery lead to a poor prognosis of HCC [7-10]. Therefore, improving the early diagnosis and searching for an effective treatment become urgent problems. To date, a few markers such as hepatomaspecific $\gamma$-glutamyl transferase, hepatoma-specific AFP, oncofetal antigen glypican-3, and member 3a of Wingless- type MMTV integration site family have been developed as specific biomarkers for HCC [11-13]. Given the unsatisfactory results for prognosis, however, it needs to explore novel markers and molecular-targets for HCC [14].

Clusterin (CLU) is a highly conserved heterodimeric disulfide-linked glyco- protein (originally named Apo-J), which is widely distributed in tissues and body fluids [15]. CLU is involved in various physiological processes, such as lipid transport, apoptosis, complements cascade, DNA repair, and cell adhesion [16-18]. The mature isoform of CLU is secretory CLU (sCLU) that mainly localizes in cytoplasm and over-expresses in a wide variety of tumors with oncogenicity $[19,20]$. Recently, abnormality of sCLU level was reported to correlate closely with HCC $[21,22]$, such as sCLU-induced epithelial-mesenchymal transition [23], chemoresistance 
or metastasis [24,25], pro-hepatocarcinogenesis activity of sCLU in vitro models, interaction with oncogenes or suppressor genes, and cancer-associated pathways [26]. However, the mechanisms of sCLU in HCC progression or effects on HCC growth in vivo still remain to be clarified. Therefore, the current study was to analyze the alteration of hepatic sCLU in HCC at different staging, and inhibition of sCLU gene transcription by specific shRNA on effects of HCC growth in vitro and in vivo.

\section{RESULTS}

\section{Hepatic sCLU expression and TNM staging of $\mathrm{HCC}$}

Hepatic sCLU expression in 40 pairs of fresh HCC- and their non-tumorous- tissues (NT) is shown in Figure 1. The sCLU expression at messenger RNA (mRNA) level was observed in cancerous or nontumorous tissues by quantitative real-time polymerase chain reaction (qRT-PCR, Figure 1A). The overall level of $\mathrm{sCLU}$ mRNA[ $\left.\log _{2}(\mathrm{HCC} / \mathrm{NT})>1\right]$ in $\mathrm{HCC}$ was $75 \%$ upregulated (30/40), 7.5\% down-regulated (3/40), and $17.5 \%$ non-changed (7/40). No significant difference at staging I was found between NT and HCC. However, the sCLU mRNA level was drastically up-regulated from staging II to IV (Figure 1B). The sCLU expressions at protein level in $60 \mathrm{HCC}$ and their NT tissues were analyzed by the tissue microarray (TMA) with immunohistochemistry. As presented in Figure 1C, the sCLU staining was mainly presented in the cytoplasm. The positive rate of sCLU expression in the HCC tissues $(73.3 \%, 44 / 60)$ was significantly higher $\left(\chi^{2}=30.033, P<0.001\right)$ than that in the NT group $(23.3 \%, 14 / 60$, Figure 1D). Moreover, the incidence of sCLU expression in HCC was $37.5 \%(3 / 8)$ at staging I, 68\% (17/25) at staging II, and 88.9\% (24/37) at staging III \& IV, respectively (Figure 1E). The levels of sCLU protein consistent with their mRNA expression were gradually up-regulation with increasing HCC staging.

\section{Clinicopathological features of high sCLU expression}

The associations between sCLU and clinicopathological parameters in TMA containing 60 HCC cases are elucidated in Table 1. Results revealed that high sCLU expression was significantly linked to poor differentiation $\left(\chi^{2}=4.651, P=0.031\right)$ and advanced TNM stage $\left(\chi^{2}=6.074, P=0.014\right)$. However, no significant associations were found between sCLU expression and patients' age, gender, AFP level, portal vein invasion, HBV infection, tumor size, liver cirrhosis, lymph node metastasis, and gross classification. Furthermore, the Kaplan-Meier survival curves with log-rank tests were performed to evaluate the prognostic value of sCLU (Figure 2). Compared with low expression of sCLU,

A

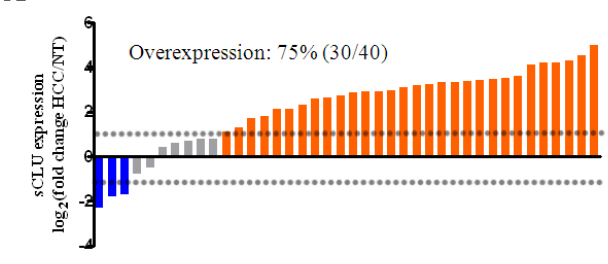

C

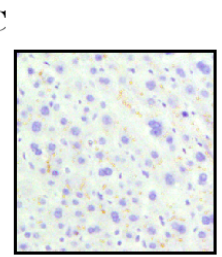

NT

\begin{tabular}{|lrrr|}
\hline \multirow{2}{*}{ Group } & \multicolumn{2}{c}{ sCLU } & Total \\
\cline { 2 - 3 } & - & $+(\%)$ & \\
HCC tissues & 16 & $44(73.3)$ & 60 \\
Non-tumorous tissues & 46 & $14(23.3)$ & 60 \\
TNM stage I & 5 & $3(37.5)$ & 8 \\
TNM stage II & 8 & $17(68.0)$ & 25 \\
TNM stage III \& IV & 3 & $24(88.9)$ & 27 \\
\hline
\end{tabular}

B

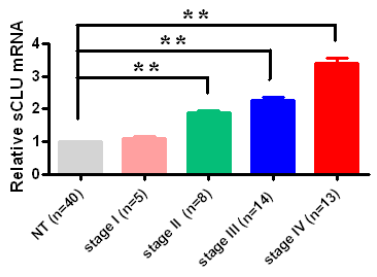

$\mathrm{HCC}$

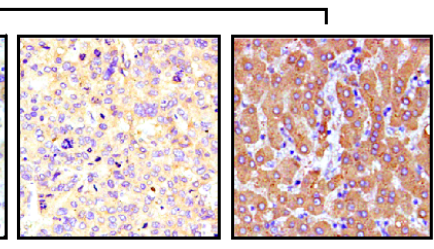

TNM stage III TNM stage IV

$\mathbf{E}$

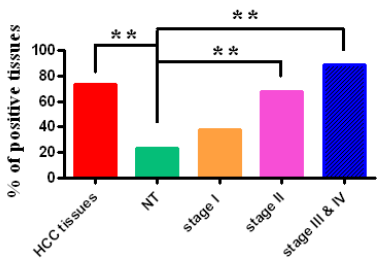

Figure 1: sCLU expression inHCC tissues. A. sCLU mRNA expression in 40 pairs of fresh HCC tissues and nontumorous tissues (NT) detected by qRT-PCR. GAPDH was used as an internal control. The dotted line represented the fold change of sCLU equal to 2 . B. sCLU mRNA expression in NT and HCC with different TNM stages determined by qRT-PCR. C. the representative sCLU immunohistochemical staining of the HCC or corresponding NT in the tissue microarrays (Original magnification $\times 400$ ); $\mathbf{D}$. the case numbers and percentages according to the sCLU expression and TNM stages of HCC; E. the bar graph summary of data presented in Figure 1D. HCC, hepatocellular carcinoma; NT, nontumorous tissues; sCLU, secretory clusterin; TNM, tumor-node-metastasis. ${ }^{* *}, P<0.01$. 
Table 1: Correlations of sCLU expression with clinical factors in HCC

\begin{tabular}{|c|c|c|c|c|}
\hline Parameters & $\mathbf{n}$ & $\begin{array}{c}\text { sCLU positive } \\
\text { n (\%) }\end{array}$ & $\chi^{2}$ value & Pvalue \\
\hline Age (years) & & & 0.135 & 0.713 \\
\hline$<50$ & 21 & $16(76.2)$ & & \\
\hline$\geq 50$ & 39 & $28(71.8)$ & & \\
\hline Gender & & & 2.532 & 0.112 \\
\hline Male & 39 & $26(66.7)$ & & \\
\hline Female & 21 & $18(85.7)$ & & \\
\hline $\operatorname{AFP}(\mu \mathrm{g} / \mathrm{L})$ & & & 0.156 & 0.693 \\
\hline$<50$ & 25 & $19(76.0)$ & & \\
\hline$\geq 50$ & 35 & $25(71.4)$ & & \\
\hline Portal vein invasion & & & 0.002 & 0.967 \\
\hline With & 19 & $14(73.7)$ & & \\
\hline Without & 41 & $30(73.2)$ & & \\
\hline HBsAg & & & 2.431 & 0.119 \\
\hline Positive & 49 & $38(77.6)$ & & \\
\hline Negative & 11 & $6(54.6)$ & & \\
\hline Tumor size & & & 0.024 & 0.876 \\
\hline$<5 \mathrm{~cm}$ & 31 & $23(74.2)$ & & \\
\hline$\geq 5 \mathrm{~cm}$ & 29 & $21(72.4)$ & & \\
\hline Liver cirrhosis & & & 0.006 & 0.936 \\
\hline With & 37 & $27(73.0)$ & & \\
\hline Without & 23 & $17(73.9)$ & & \\
\hline Lymph node metastasis & & & 2.578 & 0.108 \\
\hline With & 12 & 11(91.7) & & \\
\hline Without & 48 & $33(68.8)$ & & \\
\hline Gross classification & & & 1.091 & 0.296 \\
\hline Multifocal & 10 & $6(60.0)$ & & \\
\hline Unifocal & 50 & $38(76.0)$ & & \\
\hline Differentiation & & & 4.651 & 0.031 \\
\hline Well \& Moderate & 44 & $29(65.9)$ & & \\
\hline Poor & 16 & $15(93.8)$ & & \\
\hline TNM & & & 6.074 & 0.014 \\
\hline I \& II & 33 & $20(60.6)$ & & \\
\hline III \& IV & 27 & $24(88.9)$ & & \\
\hline
\end{tabular}

Abbreviations: AFP, $\alpha$-fetoprotein;TNM, tumor-node-metastasis; $\mathbf{S C L U}$, secretory clusterin. Boldface, $P<0.05$. 
there was a trend toward a poorer overall survival in HCC patients with positive sCLU expression $\left(\chi^{2}=5.208\right.$, $P=0.022$, Figure 2A). Besides, survival time of HCC patients with high TNM stage was significantly shorter than that of cases with low stage $\left(\chi^{2}=19.518, P<0.001\right.$, Figure 2B). Moreover, in the subset of HCC patients with TNM III \& IV stage, high sCLU expression was prone to result in a shorter survival time compared with low sCLU expression $\left(\chi^{2}=3.920, P=0.048\right.$, Figure $\left.2 \mathrm{D}\right)$; however, no obvious difference of survival time was found in patients at staging I \& II according to high or low sCLU expression $\left(\chi^{2}=0.700, P=0.403\right.$, Figure 2C).

\section{Univariate and multivariate analysis of sCLU expression}

The univariate or multivariate Cox Regression analysis identifying factors associated with 5-year survival is listed in Table 2. The univariate Cox regression analysis demonstrated that sCLU expression, gross classification, lymph node metastasis, and TNM stage were significant prognostic factors influencing survival of HCC patients. And then, the multivariate Cox regression analysis was conducted to analyze the factors above, showed the sCLU expression associated with an increased risk of death, and recommended as an independent prognostic factor for the subset of HCC patients (hazard ratio, 2.684; 95\% confidence interval: $1.072-6.722 ; P=0.035)$, along with the TNM stage (hazard ratio, 2.513; 95\% confidence interval: 1.115-5.665; $P=0.026$ ).

\section{Differences of sCLU expressions among HCC cell lines}

The expression of sCLU among different human HCC cell lines is shown in Figure 3. As shown in Figure $3 \mathrm{~A}$ and $3 \mathrm{~B}$, the expression of $\mathrm{sCLU}$ at protein or mRNA level in HCC cells (HCCLM3, MHCC97-H, SMMC-7721 and HepG2) was significantly higher than that in hepatocyte L02 cells. Furthermore, the effective shRNA-1 and a negative-control shRNA (NC-shRNA) were transfected into HCCLM3 cells, and the fluorescence
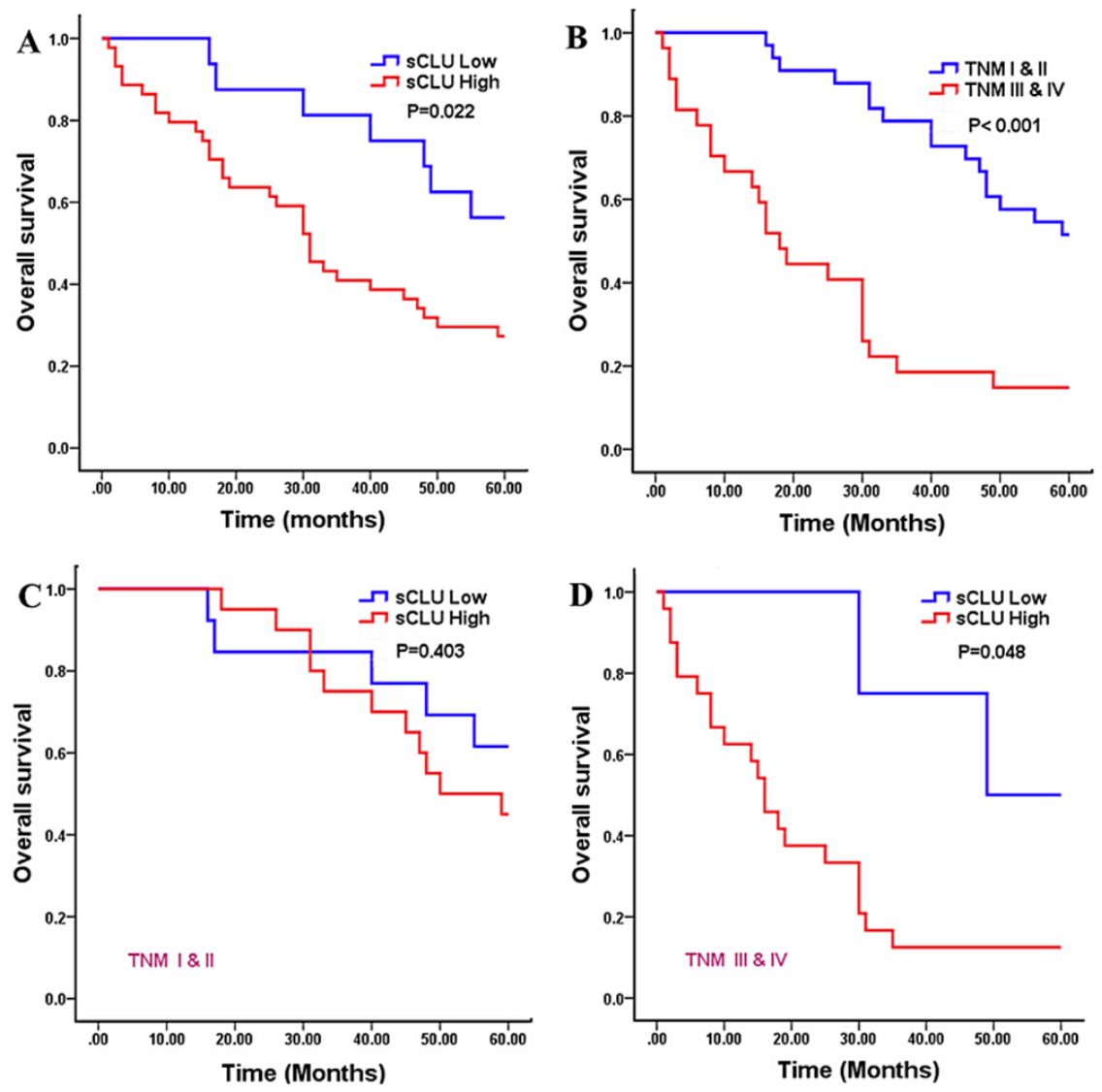

Figure 2: Kaplan-Meier analysis for overall survival of $60 \mathrm{HCC}$ patients. The survival curves were calculated according to the sCLU expression or TNM stages of HCC. Two-sided log-rank tests were performed to evaluate the statistical significance. A. the 5-year overall survival curve made according to high or low sCLU expression $(P=0.022)$; $\mathbf{B}$. the survival curve made according to high or low TNM stages $(P<0.001)$; C. the 5-year overall survival curve made according to sCLU expression in HCC patients at I $\sim$ II stages $(P=0.403)$; D. the 5-year overall survival curve made according to sCLU expression in HCC at III $\sim$ IV stages $(P=0.048)$. The red line is the higher sCLU group; and the blue line is the lower or negative sCLU group. HCC, hepatocellular carcinoma; sCLU, secretory clusterin; TNM, tumor-node-metastasis. 
photomicrographs were observed under a microscope (Figure 3C). As presented in Figure 3E, shRNA-1 could significantly decrease the expression of sCLU-mRNA in HCCLM3 (56.67\%, $q=18.19, P<0.001)$. Consistently, the sCLU protein expression was also obviously repressed in cells transfected with shRNA-1, in contrast to the control or NC-shRNA group (Figure 3D).

\section{Specific shRNA suppressed proliferation of HCC cells}

The colony formation and proliferation in HCC cells mediated by sCLU is presented in Figure 4. The colony numbers in the shRNA-1 group (105.3 \pm 22.8$)$ were significantly less than these in the control group (447.3 $\pm 34.2, q=21.88, P<0.001)$ or the NC-shRNA group (435.3 $\pm 22.5, q=21.12, P<0.001$, Figure 4A), respectively. Besides, the CCK- 8 assay showed that the proliferation of HCCLM3 cells was obviously repressed after inhibition of sCLU gene transcription by specific shRNA-1(Figure
4B). Furthermore, the protein levels of AKT and GSK-3 $\beta$, which were crucial to HCC proliferation, were detected after sCLU silencing. The total protein levels of AKT and GSK-3 $\beta$ had no obvious changes, while $\mathrm{p}-\mathrm{AKT}$ and p-GSK-3 $\beta$ expressions decreased significantly after knockdown of sCLU (Figure 4C).

\section{Silencing sCLU suppressed HCC growth in vivo}

The tumor growth and morphology are shown in Figure 5. The mice were sacrificed at the $34^{\text {th }}$ day after injection (Figure 5A). The mean weight of the xenograft tumors in the shRNA-1 group $(0.21 \pm 0.05 \mathrm{~g})$ was significantly less than that of control group $(0.8 \pm 0.07 \mathrm{~g}$, $q=20.73, P<0.001)$ and the NC-shRNA group $(0.78 \pm 0.08 \mathrm{~g}$, $q=20.22, P<0.001$; Figure 5B), respectively. Besides, growth curves of subcutaneously xenograft tumors indicated that the tumor growth of shRNA-1 group was significantly slower than that of the control and the NCshRNA group after the $18^{\text {th }}$ day (Figure $5 \mathrm{C}$ ). The sCLU

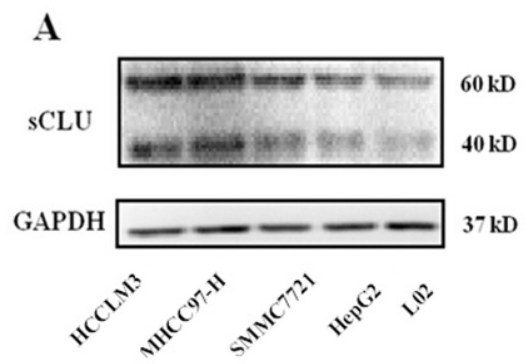

B
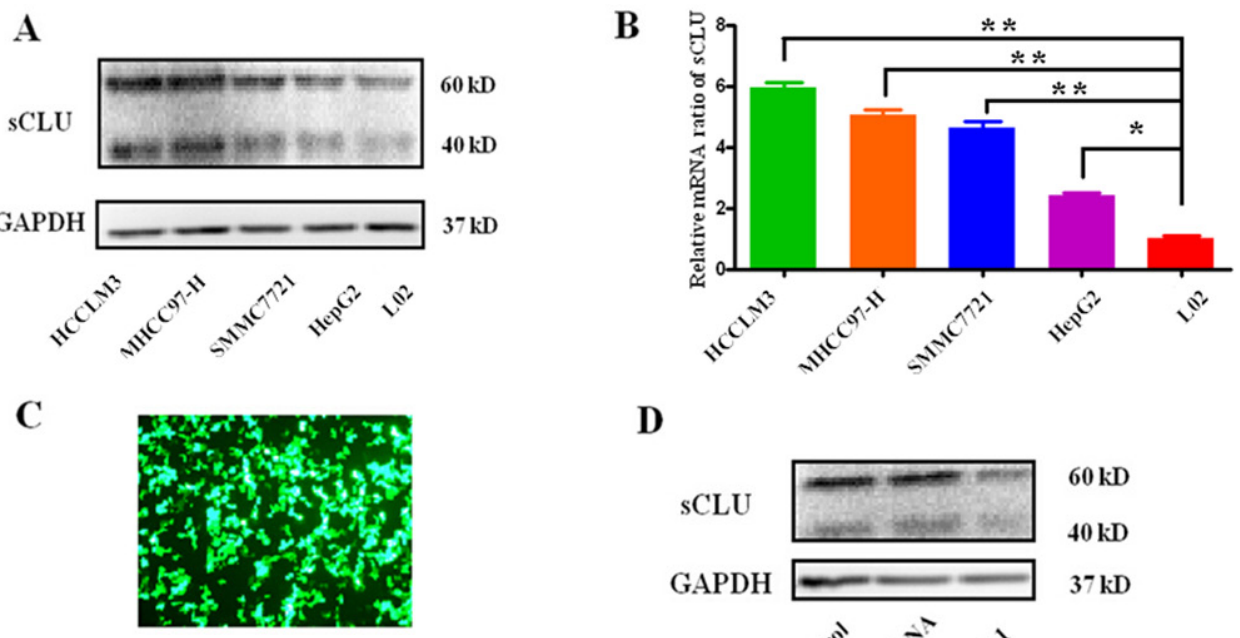

$\mathbf{D}$

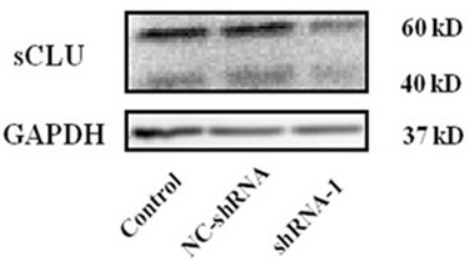

E

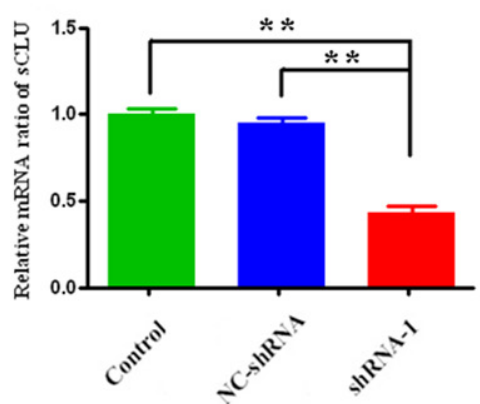

Figure 3: Expression of sCLU in HCC cells or silencing by specific shRNA. Expression of sCLU in HCCLM3, MHCC97-H, SMMC7721, HepG2, and L02 cells was detected by Western blotting at protein level or qRT-PCR at mRNA level. Then, the HCCLM3 cells were transfected with the specific shRNA to abrogate sCLU expression. A. sCLU expression among the HCC cells analyzed by the Western blotting with GAPDH as the internal control; B. sCLU mRNA expression among the HCC cells analyzed by the qRT-PCR; C. the fluorescence photomicrographs after the HCCLM3 cells transfected with specific shRNA-1; D. the comparative analysis of sCLU expression in the group with or without shRNA-1 transfection detected by the Western blotting; E. the comparative analysis of sCLU mRNA expression in the group with or without shRNA-1 transfection detected by qRT-PCR. The data were presented as means \pm SD $(n=3)$. $*, P<0.05$; **, $P<0.01$. 
Table 2: Univariate and multivariate analysis of sCLU expression in HCC tissues

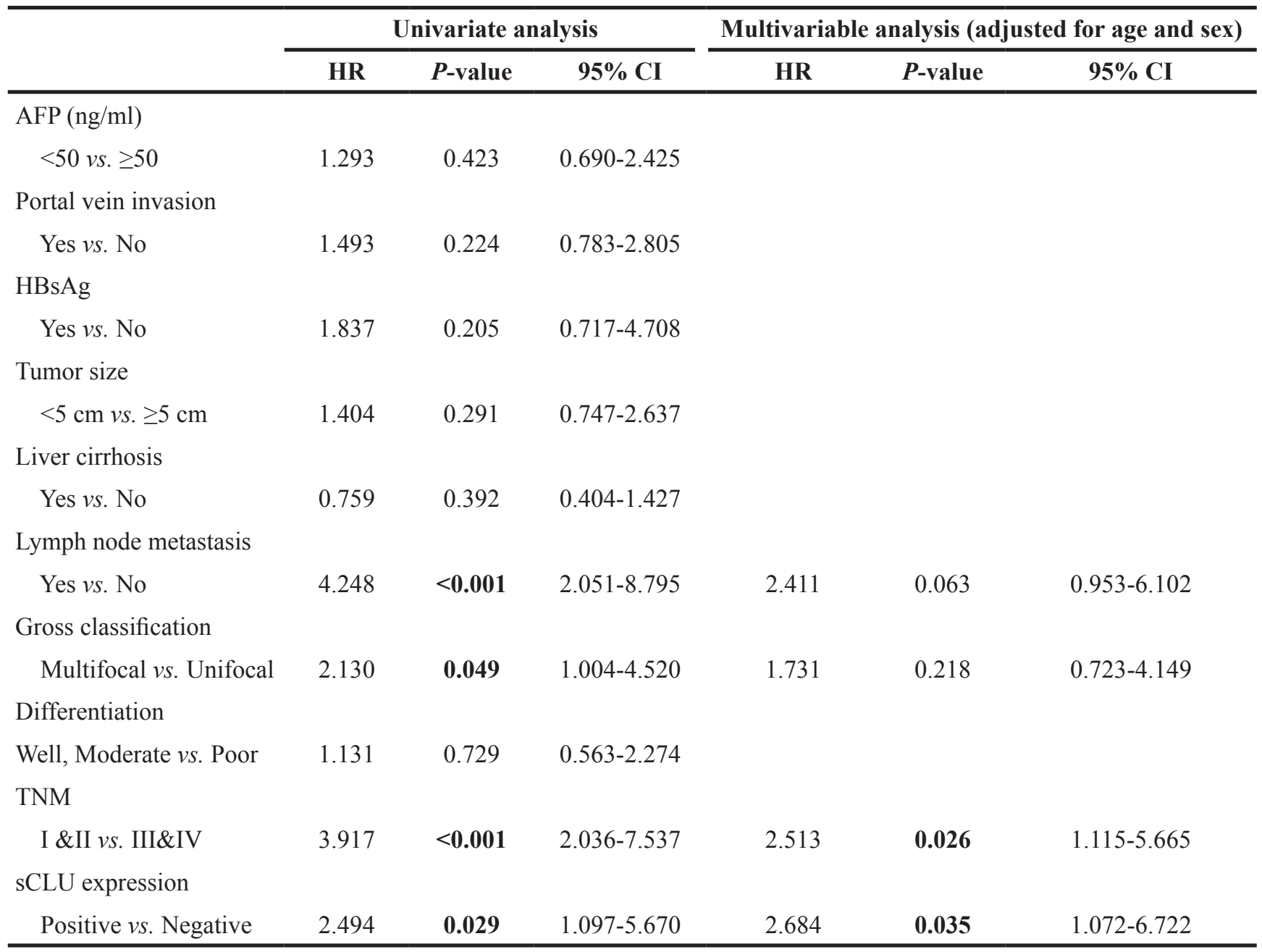

Abbreviations: CI, confidence interval; HR, hazard ratio; AFP, $\alpha$-fetoprotein; TNM,tumor-node-metastasis; sCLU, secretory clusterin. Boldface, $P<0.05$.

mRNA level in the shRNA-1 group was significantly lower than that in the control $(q=20.22, P<0.001)$ or the NC-shRNA group ( $q=20.22, P<0.001$; Figure 5D). Consistently, the sCLU protein expression in the shRNA-1 group was also lower than that in the control $(q=36.44$, $P<0.001)$ or the NC-shRNA group $(q=35.58, P<0.001)$. In addition, the tumor morphological alterations of audio-videoailable atypia among the different groups were confirmed by hematoxylin-eosin staining (H\&E). Moreover, the sCLU staining in the control or NC-shRNA group was stronger than that in the shRNA-1 group by immunohistochemistry (Figure 5E).

\section{DISCUSSION}

$\mathrm{HCC}$ is one of the most frequently diagnosed cancers in the world. Mounting reports note that HCC development is a multi-step and multi-centric process with the activation of oncogenes and inactivation of tumor supressor genes [27]. Therefore, it is of great significance to find novel and effective targets against HCC. Recent studies indicate that sCLU is over-expressed in various aggressive neoplasms and plays crucial roles in the development and progression of malignancies [28]. In the present study, the oncogenic sCLU expression at different staging of HCC progression and its application value as a molecular target for $\mathrm{HCC}$ was investigated in vitro and in vivo.

Though previous studies determined the abnormal expression of sCLU and its prognostic value in $\mathrm{HCC}$, the alteration of sCLU expression in different staging of HCC had not been explored [29]. The current data demonstrated that sCLU was over-expressed in HCC tissues at mRNA or protein level, which was significantly higher than that in the corresponding nontumorous tissues. Notably, our study found that sCLU mRNA and protein expression presented a gradual upregulation in the HCC cases from low clinical stage to high stage. Besides, hepatic sCLU increased drastically from the group of stage I to II, indicating a positive role in early progression of HCC. Moreover, high sCLU expression was significantly associated with poor differentiation, advanced TNM stage, and short survival 
A

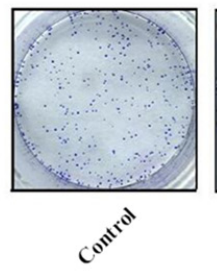

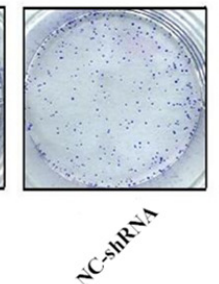

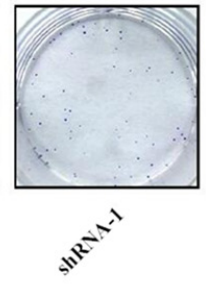

B

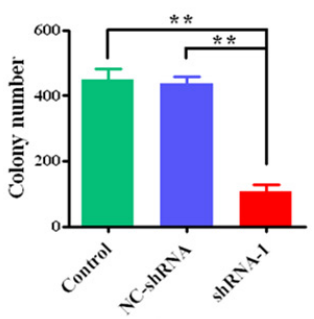

C

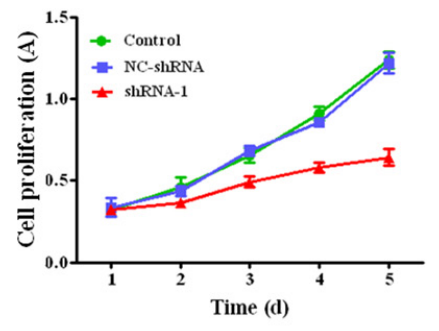

D

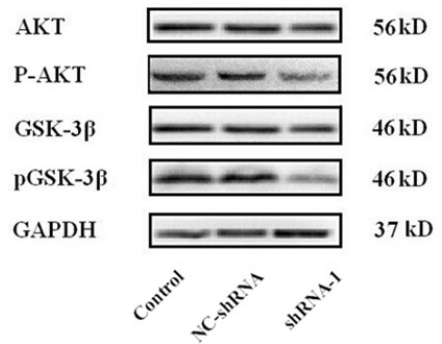

Figure 4: Silencing sCLU on effects of colony formation and cell proliferation. A. the colony formation assay of HCC cells, and the representative micrographs from the Giemsa-stained colonies formed by HCCLM3 cells with transfection of shRNA-1, NCshRNA or without transfection; B. the comparative analysis of the micrograph quantification results from the Giemsa-stained HCCLM3 cells transfected with shRNA-1, NC-shRNA or control group $(\mathrm{n}=3)$; C. the viability of HCCLM3 cells transfected with the shRNA-1, NC-shRNA or without transfection determined by CCK-8 assay on days 1 to 5 ; D. the levels of AKT, phosphorylated-AKT, GSK-3 $\beta$, and phosphorylated-GSK-3 $\beta$ expressions detected by Western blotting. The data were presented as means $\pm \mathrm{SD}(\mathrm{n}=3) . * *, P<0.01$.

A

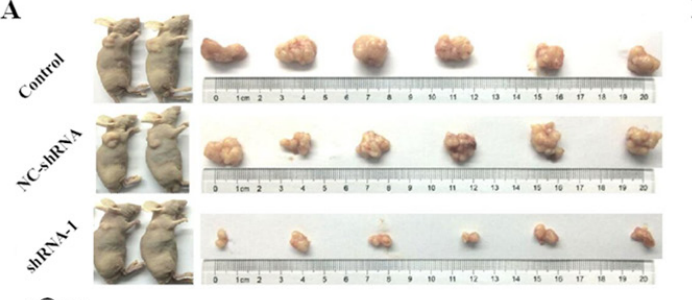

C

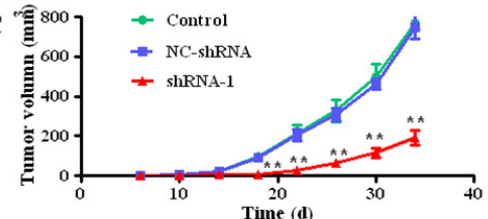

D
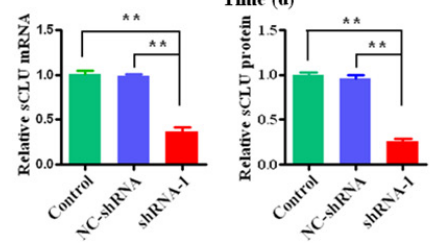

$\mathbf{E}$
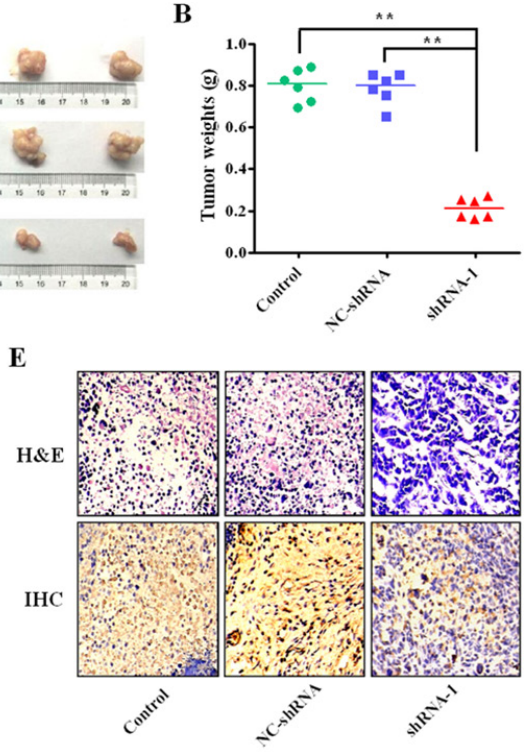

Figure 5: Intervening sCLU gene transcription on effect of xenograft growth. HCCLM3 cells transfected with shRNA-1, NC-shRNA or without transfection were injected into nude mice to observe the formation of tumor. A. the representative photographs of the nude mice and corresponding dissected tumors from each group; B. the mean tumor weights in nude mice subcutaneously inoculated with the HCCLM3 cells transfected with shRNA-1 and NC-shRNA or without transfection as control; $\mathbf{C}$. the growth curves of the xenograft tumors among the different groups, and the tumor volumes were measured every 4 days; $\mathbf{D}$. the relative expression of sCLU mRNA in each group was detected by the qRT-PCR with comparative cycle threshold (Ct) method. The relative sCLU expression at protein level was calculated according to the ratio from tissue sCLU concentration to total protein by ELISA analysis; E. the patho-histology or the alteration of sCLU expression in the resected tumor tissues were examined by the H\&E or immunohistochemical staining, respectively (Original magnification $\times 400$ ). The data were presented as means $\pm \mathrm{SD}$ of duplicate experiments. H\&E, Hematoxylin and Eosin; IHC, immunohistochemistry. **, $P<0.01$. 
time of HCC patients, suggesting that sCLU might promote the early progression of HCC and should be an early molecular biomarker for HCC.

Increasing evidence note that sCLU overexpression may promote proliferation of cancer cells [30, 31]. This study further conducted related assays on HCCLM3 cells to explore the potential functions of sCLU. Obvious inhibition of colony formation and proliferation was observed in HCCLM3 cells after knock-down of sCLU. Certain signal pathways might be involved in sCLU-mediated proliferation. Activation of AKT could deteriorate the activity of GSK-3 $\beta$, subsequently up-regulating the oncogenes of the downstream, and ultimately promoting the proliferation of malignant cells [32-34]. Notably, our study discovered that silencing sCLU decreased phosphorylated AKT expression (active form) and phosphorylated GSK-3 $\beta$ expression (inactive form), indicating that AKT inactivation and GSK-3 $\beta$ activation might relate to proliferation of HCC cells.

Mounting studies demonstrate that sCLU is involved in the development of various malignancies. Abrogating sCLU expression could significantly inhibit the growth of tumor derived from cancer cells $[35,36]$. Conversely, sCLU-overexpression resulted in a fast growth in the orthotopic primary tumor [37]. Then, our study further explored the possible hepatocarcinogenesis function of sCLU in vivo. Obviously, the xenograft tumors formed by sCLU-silenced HCC cells grew much slower than the control group. Besides, it was validated that the sCLU expression in small xenograft tumor was significantly lower than that in large size tumor. Given the in vivo findings, sCLU may be considered to participate in the tumorigenicity of HCC.

In conclusion, the up-regulation of sCLU expression at early staging of $\mathrm{HCC}$ is considered to promote tumor development and exacerbate the survival of HCC patients, which may be related to the phosphorylation of AKT/ GSK-3 $\beta$. Targeted therapy offers an effective option for non-surgical HCC management, but it remains to be a challenge due to lack of specific targets. Silencing sCLU provides a new mechanism insight into molecular-targeted therapy for its inhibition of HCC growth. Admittedly, to clarify the exact role of sCLU more roundly and convincingly, larger number of clinical cases and delicate experiments could be adopted in further studies. It is expected that inhibiting sCLU gene transcription plus multi-targeting strategies should be explored for HCC therapy [38].

\section{MATERIALS AND METHODS}

\section{Ethics statement}

The study protocol was approved by the Research Ethics Committee of Affiliated Hospital of Nantong University (approval number: TDFY2012-1) and performed according to the World Medical Association
Declaration of Helsinki [39]. Written or verbal consent was required from each patient involved in the study.

\section{Liver tissues}

A total of 60 primary HCC- and their selfmatched NT tissues were collected from HCC patients who received hepatectomy at the Affiliated Hospital of Nantong University, Nantong, China from Jan 2008 to Jan 2010. The diagnosis of HCC was confirmed histologically in each patient, and no patient underwent chemotherapy or radiotherapy before the surgery. Clinical information was obtained from medical records and included a 5-year post-surgery follow-up period. For each case, patient's gender, age, TNM stage, tumor size, differentiation, HBsAg infection, portal vein invasion, and lymph node metastasis status were recorded. In addition, 40 pairs of fresh HCC tissues and matched nontumorous tissues were obtained from the patients who underwent hepatectomy at the Affiliated Hospital of Nantong University from Sept 2012 to Sept 2014. After surgery, the liver tissues were immediately frozen at $-80^{\circ} \mathrm{C}$ for further RNA isolation. The diagnosis of HCC, cirrhosis, and viral hepatitis was based on the criteria proposed by the Ministry of Health of the People's Republic of China and the Chinese National Viral Hepatitis Meeting, respectively.

\section{Tissue microarray (TMA) and immunohistochemistry (IHC)}

TMA chip containing 60 cancerous- and matched NT- tissues were constructed by the Outdo Biotech Company (Shanghai, China). IHC assay was performed by using the Autostainer Universal Staining System (LabVision, USA) as a previous study [40]. In brief, the TMA sections were deparaffinized in xylene and quenched by $0.3 \%(\mathrm{~V} / \mathrm{V})$ hydrogen peroxide in methanol. After treating with citrate buffer and endogenous peroxidase, the slides were blocked in 10\% goat serum to avoid nonspecific staining. And then slides were incubated overnight with mouse anti-human sCLU monoclonal antibody (1:200, Santa Cruz, USA) at $4^{\circ} \mathrm{C}$. Following washing in phosphate buffered saline (PBS) for 3 times, the slides were incubated with horse reddish peroxidaseconjugated goat anti-mouse antibody (1:1000, DAKO, USA) at room temperature for $30 \mathrm{~min}$. Finally, the TMA sections were visualized with diaminobenzine and counterstained with hematoxylin (Kem-En-Tec Diagnostics, Denmark).

\section{Evaluation of sCLU expression in HCC tissues}

The IHC staining results of sCLU expression in TMA section were evaluated independently by two experienced pathologists using a semi-quantitative score system. IHC score of each sample was defined as the percentage sum of positive staining level $(0,1,2$, and 3$)$ 
and the staining intensity $(0,1,2$, and 3$)$. According to the IHC score, the cancerous tissues were divided into two groups: the low sCLU expression with $0 \sim 2$ scores and high expression with 3 6 scores. Given the differences between duplicate tissue scores, the higher score was taken as the final score.

\section{Specific shRNA plasmid construction}

The shRNAs targeting different sites of human sCLU gene according to the GenBank ID 001831.3 were designed and synthesized by Biomics Company (Nantong, China). And the most effective shRNA silencing sCLU was screened as the previously described [25]. NC-shRNA or effective shRNA-1 was constructed into pGPU6/GFP/Neo vectors, and inserted sequences were verified by sequencing. Sequences: shRNA-1, 5'-GTAAGTACGTCAATAAGGA-3'; and NC-shRNA, 5'-TTCTCCGAACGTGTCACGT-3'.

\section{Cell culture and transfection}

Human hepatoma HCCLM3, SMMC7721, MHCC97-H, HepG2, and hepatocyte L02 cells were purchased from Chinese Academy of Sciences (Shanghai, China) and cultured in Dulbecco's modified Eagle's medium (DMEM, KeyGen Biotech, Nanjing, China) with $10 \%$ fetal bovine serum (FBS, Gibco, USA) at $37^{\circ} \mathrm{C}$ in a humidified incubator containing $5 \% \mathrm{CO}_{2}$. Specific plasmids were transfected into cells with GenJet ${ }^{\mathrm{TM}}$ DNA in Vitro Transfection Reagent (SignaGen, USA) according to the manufacturer's instructions. Briefly, cells were planted in 6-well plates at $24 \mathrm{~h}$ prior to transfection. Fresh culture medium was replaced in each well at $1 \mathrm{~h}$ before transfection. Then, $200 \mu \mathrm{L}$ of DMEM mixture containing DNA and GenJet ${ }^{\mathrm{TM}}$ (1:3) was cautiously added into each well. After incubating for $12 \mathrm{~h}$, the medium was replaced by fresh complete medium. Finally, the transfection efficiency was observed by the fluorescence microscope.

\section{Colony formation analysis}

Human hepatoma cells $\left(1 \times 10^{3}\right.$ cells/well $)$ transfected with NC-shRNA, shRNA-1 or without transfection were seeded in 6-well plates and continuously incubated for 10 days. During the indicated time, the culture medium was replaced every 3 days. Then, cells were washed by phosphate buffer saline (PBS), fixed by methanol for 30 min, stained with Giemsa (Beyotime, Jiangsu, China). The single colony with more than 50 cells was counted under a stereomicroscope. The experiment was conducted in triplicate.

\section{Proliferation assay}

Cell proliferation assay was detected by the Cell Counting Kit 8 (CCK-8, Beyotime, Jiangsu, China) according to the manufacturer's instructions. In brief, human hepatoma cells were plated in 96 -well plates at a density of $5 \times 10^{3}$ cells per well. After $24 \mathrm{~h}$, the cells were transfected with NC-shRNA, shRNA-1 or without transfection. At the followed post-transfection time (1, $2,3,4$, or 5 days), the cells was incubated in medium containing CCK- 8 reagent for $4 \mathrm{~h}$. After that, the absorbance at $450 \mathrm{~nm}$ was measured by using a microplate reader. The assays were performed in triplicate.

\section{RNA isolation and qRT-PCR}

Total RNA was isolated from tissues and cells with Trizol reagent (Invitrogen, USA) according to the manufacturer's instructions. Equal amounts of RNA were reverse transcribed into cDNA using the RevertAid ${ }^{\mathrm{TM}}$ First Strand cDNA Synthesis Kit (MBI Fermentas, CA). qRT-PCR was conducted by SYBR $®$ Premix Ex TaqTMII (TaKaRa, Dalian, China) with the manufacturer's instructions. Glyceraldehyde-3-phosphate dehydrogenase (GAPDH) was used as an internal control, and the melting curves were detected for the amplification specificity. The messenger RNA (mRNA) value was calculated based on the $\mathrm{Ct}$ values using the $2^{-\Delta \Delta \mathrm{Ct}}$ method $[\Delta \mathrm{Ct}$ $\left.=\mathrm{Ct}_{\text {target gene }}-\mathrm{Ct}_{\mathrm{GAPDH}}\right]$. The primers were as follows: sCLU, F: 3'-ATCACTGTGACGGTCCCTGTA-5', and R: 3'-TCACTCCTCCCGGTGCTT-5'; GAPDH, F: 3'-CAAGGTCATCCATGACAACTTTG-5', and R: 3'-GTCCACCACCCTGTTGCTGTAG-5'.

\section{Western blotting}

Protein samples collected by sodium dodecyl sulfate (SDS) cell lysis buffer (Beyotime, Nanjing, China) were separated by SDS-polyacrylamide gel electro- phoresis for $1.5 \mathrm{~h}$, and then transferred onto polyvinylidene fluoride membranes after $2 \mathrm{~h}$. Following blocking for $2.5 \mathrm{~h}$, the membranes were incubated in the primary antibody diluents overnight. Moreover, after washing with tris-buffered saline with tween (TBST), membranes were incubated in the secondary antibody diluents for $2 \mathrm{~h}$. Finally, the samples were detected by electrochemiluminescence (ECL) kit (Millipore, USA). Antibodies were diluted as follows: GAPDH, AKT and GSK-3 $\beta$ (1:1000; Cell Signaling, USA); sCLU (1:500; Santa Cruz, USA); phos-AKT and phos-GSK-3 $\beta$ (1:1000; Abcam, USA); IgG horseradish peroxidase conjugate (1:1000; Univ-bio, Nanjing, China).

\section{Xenograft tumor models}

All procedures on mice were performed according to the Guidelines for the Experimental Animals and approved by the Animal Care and Use Committee of Nantong University. The 6 weeks old BALB/c nude mice $(n=18)$ were provided by the Experimental Animal Center of Nantong University, and randomly assigned into 
three groups (sCLU-shRNA, NC-shRNA, and control). Briefly, hepatoma cells $\left(2 \times 10^{7}\right.$ cells $)$ that were stably transfected with sCLU-shRNA, NC-shRNA or without transfection were suspended in $200 \mu \mathrm{L}$ DMEM and injected subcutaneously into the right scapular area of nude mice. Tumor size was measured using calipers every 4 days. The tumor volume was calculated as follows: the tumor volume $\left(\mathrm{mm}^{3}\right)=0.5\left(\right.$ length $\times$ width $\left.^{2}\right)$. The animals were sacrificed at the end of 5 weeks after injection. The xenograft tumors were dissected and fixed in $4 \%$ paraformaldehyde, embedded in paraffin, deparaffinized in xylene, and dehydrated in a gradient of ethanol solutions. Finally, histopathological examination was performed with the Hematoxylin \& Eosin (H\&E) staining.

\section{Protein extraction from xenograft tumors}

Total proteins were extracted from the xenograft tumors of different groups by ice-cold radio immunoprecipitation assay lysis buffer (Beyotime, Shanghai, China). After centrifuged at 12,000 rpm for $30 \mathrm{~min}$, the lysate supernatants were harvested. The total protein levels were measured by the Bicinchonininc Acid Protein Assay Kit (Beyotime, Shanghai, China) according to the manufacturer's instructions.

\section{ELISA analysis}

Furthermore, protein level of sCLU was detected by the sCLU Enzyme-Linked Immunosorbent Assay (ELISA) Kit (Boster, Wuhan, China). In brief, $100 \mu \mathrm{L}$ lysate of each group was added into indicated wells and incubated at $37^{\circ} \mathrm{C}$ for $2 \mathrm{~h}$. Then $100 \mu \mathrm{L}$ of the sCLU antibody solution and the avidin-biotin complex solution was added in sequence. Contents of wells were aspirated and washed 5 times. After that, $100 \mu \mathrm{L}$ of the tetramethyl benzidine development solution and $90 \mu \mathrm{L}$ of Stop solution were added to appropriate wells to stop reaction. Finally, the values at $\mathrm{A}_{450} \mathrm{~nm}$ of duplicate samples were detected with a microplate reader (MD, USA).

\section{Statistical methods}

Data were presented as means \pm standard deviation (SD), and analyzed by using the SPSS19.0 Software. The relationships between clinical features and sCLU expression were determined by the $\chi^{2}$ test. Kaplan-Meier analysis with a log-rank test was performed to calculate the survival curves. The univariate and multivariate Cox regression analyses were conducted to evaluate the prognostic value. Comparisons among the Control, NC-shRNA, and shRNA-1 groups were performed by Student's Newman-Keuls (SNK) comparison. $P<0.05$ was considered to be statistically significant.

\section{Abbreviations}

AFP, alpha-fetoprotein; ELISA, enzymelinked immunosorbent assay; HCC, hepatocellular carcinoma; H\&E, Hematoxylin and Eosin; IHC, immunohistochemistry; PBS, phosphate buffer saline; sCLU, secretory clusterin; TMA, tissue microarray.

\section{ACKNOWLEDGMENTS}

This work was supported by grants-in-aid from Projects of the Chinese National Natural Science Foundation (No: 81673241, 81200634), the Jiangsu Health Projects (2014-YY-028, 2014-WSN078, and BE2016698), the Science Foundation of Nantong Health and Family Planning Commission (WQ2016083), and the International S.\&T. Cooperation Program(2013DFA32150) of China.

\section{CONFLICTS OF INTEREST}

The authors declare none conflicts of interests.

\section{REFERENCES}

1. Bruix J, Reig M, Sherman M. Evidence-based diagnosis, staging, and treatment of patients with hepatocellular carcinoma. Gastroenterology. 2016; 150: 835-853.

2. Forner A, Llovet JM, Bruix J. Hepatocellular carcinoma. Lancet. 2012; 379:1245-1255.

3. Pascual S, Herrera I, Irurzun J. New advances in hepatocellular carcinoma. World J Hepatol. 2016; 8:421-438

4. El-Serag HB. Epidemiology of viral hepatitis and hepatocellular carcinoma. Gastroenterol. 2012; 142:1264-1273.

5. Maluccio M, Covey A. Recent progress in understanding, diagnosing, and treating hepatocellular carcinoma. CA Cancer J Clin. 2012; 62:394-399.

6. Asim M, Sarma MP, Thayumanavan L, Kar P. Role ofaflatoxin B1 as a risk for primary liver cancer in north Indian population. Clin Biochem. 2011; 44:1235-1240.

7. Hollebecque A, Malka D, Ferte C, Ducreux M, Boige V. Systemic treatment of advanced hepatocellular carcinoma: from disillusions to new horizons. Eur J Cancer. 2015; $51: 327-339$

8. Akoad ME, Pomfret EA. Surgical resection and liver transplantation for hepato- cellular carcinoma. Clin Liver Dis. 2015; 19:381-399.

9. Kalyan A, Nimeiri H, Kulik L. Systemic therapy of hepatocellular carcinoma: current and promising. Clin Liver Dis. 2015; 19:421-432. 
10. Moriguchi M, Umemura A, Itoh Y. Current status and future prospects of chemo- therapy for advanced hepatocellular carcinoma. Clin J Gastroenterol. 2016; 9:184-190.

11. Wang L, Yao M, Dong Z, Zhang Y, Yao D. Circulating specific biomarkers in diagnosis of hepatocellular carcinoma and its metastasis monitoring. Tumour Biol. 2014; 35:9-20.

12. Wang L, Pan LH, Yao M, Cai Y, Dong ZZ, Yao DF. Expression of oncofetal antigen glypican-3 associates significantly with poor prognosis in HBV-related hepatocellular carcinoma. Oncotarget. 2016; 7:4215042158. doi: 10.18632/oncotarget.9892.

13. Pan LH, Yao M, Zheng WJ, Gu JJ, Yang XL, Qiu LW, Cai Y, Wu W, Yao DF. Abnormality of Wnt3a expression as novel specific biomarker for diagnosis and differentiation of hepatocellular carcinoma. Tumour Biol. 2016; 37:5561-5568.

14. Takami T, Yamasaki T, Saeki I, Matsumoto T, Suehiro Y, Sakaida I. Supportive therapies for prevention of hepatocellular carcinoma recurrence and preservation of liver function. World J Gastroenterol. 2016; 22:7252-7263.

15. Novinec M, Lenarčič B, Baici A. Clusterin is a specific stabilizer and liberator of extracellular cathepsin K. FEBSLett. 2012; 586: 1062-1066.

16. Trougakos IP, Djeu JY, Gonos ES, Boothman DA. Advances and challenges in basic and translational research on clusterin. Cancer Res. 2009; 69:403-406.

17. Prochnow H, Gollan R, Rohne P, Hassemer M, KochBrandt C, BaiersdörferM. Non-secreted clusterin isoforms are translated in rare amounts from distinct human mRNA variants and do not affect Bax-mediated apoptosis or the NF- $\mathrm{B}$ signaling pathway. PLoS One. 2013; 8:e75303.

18. Muhammad LA, Saad F. The role of clusterin in prostate cancer: treatment resistance and potential as a therapeutic target. Expert Rev Anticancer Ther. 2015; 15:1049-1061.

19. Luo X, Suzuki M, Ghandhi SA, Amundson SA, Boothman DA. ATM regulates insulin-like growth factor 1-secretory clusterin (IGF-1-sCLU) expression that protects cells against senescence. PLoS One. 2014; 9:e99983.

20. Koltai T. Clusterin: a key player in cancer chemoresistance and its inhibition. Onco Targets Ther. 2014; 7:447-456.

21. Zhang J, Miao C, Xu A, Zhao K, Qin Z, Li X, Liang C, Hua Y, Chen W, Zhang C, Liu Y, Su S, Wang Z, Liu B. Prognostic role of secretory clusterin in multiple human malignant neoplasms: a meta-analysis of 26 immunohistochemistry studies. PLoS One. 2016; 11:e0161150.

22. Zheng WJ, Yao M, Sai WL, Qian Q, Pan LH, Qiu LW, Huang JF, Wu W, Yao DF. Diagnostic and prognostic significance of secretory clusterin expression in patients with hepatocellular carcinoma. Tumour Biol. 2016; 37:999-1008

23. Wang C, Jiang K, Kang X, Gao D, Sun C, Li Y, Sun L, Zhang S, Liu X, Wu W, Yang P, Guo K, Liu Y. Tumorderived secretory clusterin induces epithelial-mesenchymal transition and facilitates hepatocellular carcinoma metastasis. Int J Biochem Cell Biol. 2012; 44:2308-2320.

24. Xiu P, Dong X, Dong X, Xu Z, Zhu H, Liu F, Wei Z, Zhai B, Kanwar JR, Jiang H, Li J, Sun X. Secretory clusterin contributes to oxaliplatin resistance by activating Akt pathway in hepatocellular carcinoma. Cancer Sci. 2013; 104:375-382.

25. Zheng WJ, Sai WL, Yao M, Gu H, Yao Y, Qian Q, Yao DF. Silencing clusterin gene transcription on effects of multidrug resistance reversing of human hepatoma HepG2/ ADM cells. Tumour Biol. 2015; 36:3995-4003.

26. Wang C, Jiang K, Gao D, Kang X, Sun C, Zhang Q, Li Y, Sun L, Zhang S, Guo K, Liu Y. Clusterin protects hepatocellular carcinoma cells from endoplasmic reticulum stress induced apoptosis through GRP78. PLoS One. 2013; 8:e55981.

27. Llovet JM, Zucman-Rossi J, Pikarsky E, Sangro B, Schwartz M, Sherman M, Gores G. Hepatocellular carcinoma. Nat Rev Dis Primers. 2016; 2:16018-16027.

28. Choi B, Kang SS, Kang SW, Min BH, Lee EJ, Song DH, Kim SM, Song Y, Yoon SY, Chang EJ. Secretory clusterin inhibits osteoclastogenesis by attenuating M-CSFdependent osteoclast precursor cell proliferation. Biochem Biophys Res Commun. 2014; 450:105-109.

29. Wang C, Jin G, Jin H, Wang N, Luo Q, Zhang Y, Gao D, Jiang K, Gu D, Shen Q, Huo X, Hu F, Ge T, et al. Clusterin facilitates metastasis by EIF3I/Akt/MMP13 signaling in hepatocellular carcinoma. Oncotarget. 2015; 6:2903-2916. doi: 10.18632/oncotarget.3093.

30. Fu Y, Lai Y, Liu J, Liu X, You Z, Yang G. Lentivirusmediated shRNA interference of clusterin blocks proliferation, motility, invasion and cell cycle in the ovarian cancer cells. J Ovarian Res. 2015; 8:59-67.

31. Shi H, Deng JH, Wang Z, Cao KY, Zhou L, Wan H. Knockdown of clusterin inhibits the growth and migration of renal carcinoma cells and leads to differential gene expression. Mol Med Rep. 2013; 8:35-40.

32. Lin L, Fang Z, Lin H, You H, Wang J, Su Y, Wang F, Zhang ZY. Depletion of Cks1 and Cks2 expression compromises cell proliferation and enhance chemo- therapy-induced apoptosis in HepG2 cells. Oncol Rep. 2016; 35:26-32.

33. Ye X, Guo Y, Zhang Q, Chen W, Hua X, Liu W, Yang Y, Chen G. $\beta$ Klotho suppresses tumor growth in hepatocellular carcinoma by regulating Akt/GSK-3 $\beta /$ cyclin D1 signaling pathway. PLoS One. 2013; 8:e55615.

34. Liao K, Li J, Wang Z. Dihydroartemisinin inhibits cell proliferation via $\mathrm{AKT} / \mathrm{GSK} 3 \beta /$ cyclinD1 pathway and induces apoptosis in A549 lung cancer cells. Int J Clin Exp Pathol. 2014; 7:8684-8691.

35. Chuma M, Terashita K, Sakamoto N. New molecularly targeted therapies against advanced hepatocellular carcinoma: From molecular pathogenesis to clinical trials and future directions. Hepatol Res. 2015; 45:E1-E11. 
36. Lee JY, Kim HJ, Rho SB, Lee SH. eIF3f reduces tumor growth by directly interrupting clusterin with anti-apoptotic property in cancer cells. Oncotarget. 2016; 7:18541-18557. doi: 10.18632/oncotarget.8105.

37. Flanagan L, Whyte L, Chatterjee N, Tenniswood M. Effects of clusterin over-expression on metastatic progression and therapy in breast cancer. BMC Cancer. 2010; 10:107.

38. Yao M, Wang L, Qiu L, Qian Q, Yao D. Encouraging microRNA-based thera- peutic strategies for hepatocellular carcinoma. Anticancer Agents Med Chem. 2015; $15: 453-460$
39. General Assembly of the World Medical Association. World Medical Association Declaration of Helsinki: ethical principles for medical research involving human subjects. Am Coll Dent. 2014; 81:8-14.

40. Pan LH, Yao M, Cai Y, Gu JJ, Yang XL, Wang L, Yao DF. Oncogenic Wnt3a expression as an estimable prognostic marker for hepatocellular carcinoma. World J Gastroenterol. 2016; 22:3829-3836. 